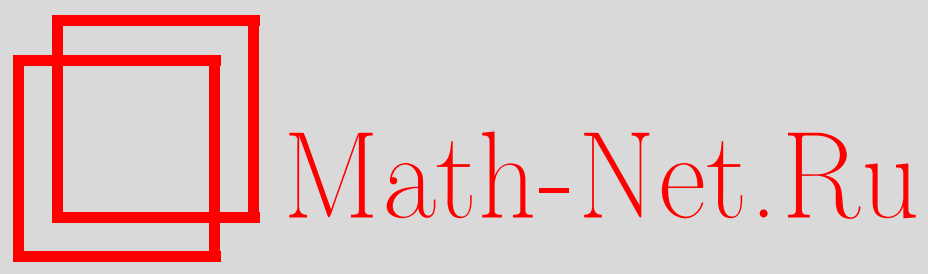

Б. А. Погорелов, М. А. Пудовкина, О расстояниях от подстановок до объединения всех импримитивных групп с равными параметрами систем импримитивности, Дискрет. матем., 2014, том 26, выпуск 1, 103-117

DOI: https://doi.org/10.4213/dm1271

Использование Общероссийского математического портала Math-Net.Ru подразумевает, что вы прочитали и согласны с пользовательским соглашением http://www.mathnet.ru/rus/agreement

Параметры загрузки:

IP: 54.157 .27 .8

26 апреля 2023 г., 12:06:00

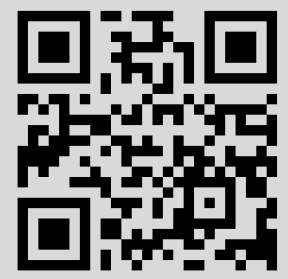




\title{
О расстояниях от подстановок до объединения всех импримитивных групп с равными параметрами систем импримитивности
}

\author{
() 2014 г. Б. А. Погорелов, М. А. Пудовкина ${ }^{2}$
}

\begin{abstract}
В работе исследуются расстояния Хемминга от подстановок степени $n=w r$ до объединения всех групп $S_{w} \prec S_{r}$ с фиксированными параметрами $w, r$ и объединения всех таких групп с произвольными нетривиальными параметрами $w, r$. Приведены расстояния от известных $s$-боксов до некоторых сплетений групп.
\end{abstract}

Ключевые слова: система импримитивности, линейные структуры, метрические пространства, расстояния от подстановки до импримитивной группы, сплетение.

\section{1. Введение}

В дискретной математике, теории кодирования и криптографии ряд задач сводится к нахождению расстояния от преобразований, заданных на конечном множестве $X$, до некоторого подмножества $H$ симметрической группы $S(X)$ (или сокращённо $S_{n}=S(X)$ при $\left.n=|X|\right)$. Например, в криптографии $H$ может быть подмножеством множества всех аффинных преобразований. Кроме того, имеется немало работ, посвящённых построению классов функций, максимально далеких от множества всех аффинных функций, или оценивающих расстояния между классами функций. Зачастую эти задачи является довольно сложными. Так, до сих пор не описано множество всех бент-функций, не известно их число. Данные вопросы вызывают большой интерес в теории кодирования и криптографии. Например, чем меньше расстояние между координатными функциями раундовых преобразований и множеством всех аффинных функций, тем менее стойка, вообще говоря, шифрсистема к линейному методу. По аналогии вместо множества всех функций можно рассматривать симметрическую группу $S_{n}$, а в качестве множества всех аффинных функций - сплетение симметрических групп $S_{w} \prec S_{r}$, т. е. множество всех подстановок, сохраняющих некоторое разбиение $W$ множества $X$ на равномощные подмножества (разбиение $W$

\footnotetext{
${ }^{1}$ Место работы: Академия криптографии Российской Федерации e-mail: pogorelow.b@yandex.ru

${ }^{2}$ Место работы: Национальный исследовательский ядерный университет (МИФИ) e-mail: maricap@rambler.ru
} 
называется системой импримитивности). Поэтому к шифрсистемам с раундовыми функциями, принадлежащими некоторому сплетению симметрических групп, естественным образом применяется, например, аналог метода гомоморфизмов [1]. Если раундовые функции не принадлежат некоторому сплетению симметрических групп, то при применении метода приближённых гомоморфизмов [2] оценивается расстояние (например, относительно метрики Хемминга) от функции зашифрования до $S_{w}<S_{r}$, а затем фактически рассматривается её приближение подстановками из $S_{w} \prec S_{r}$. Кроме того, как было показано в [3], в некоторых случаях в разностном методе оценивается расстояние от функции зашифрования до смежного класса симметрической группы по некоторому сплетению симметрических групп.

В [4] рассматривались расстояния до сплетения групп $S_{w}$ 2 $S_{r}$ с фиксированной системой импримитивности $W$. В этом случае каждой подстановке ставится в соответствие матрица, характеризующая удалённость данной подстановки от группы $S_{w} \imath S_{r}$. Через коэффициенты этой матрицы получено выражение расстояния от произвольной подстановки до группы $S_{w} 2 S_{r}$. Описаны подстановки, максимально далекие от сплетения групп $S_{w}$ 乙 $S_{r}$ с фиксированной системой импримитивности.

В настоящей работе рассматривается расстояние от подстановок до множества $G$, являющегося:

- объединением $\mathrm{IG}_{(w, r)}$ сплетений симметрических групп, т. е. фиксированы только параметры $w, r$ системы импримитивности, но не конкретная система импримитивности;

- объединением всевозможных нетривиальных сплетений симметрических групп, т. е. система импримитивности может быть любой нетривиальной.

Близость подстановок $g, h \in S_{n}$ будет измеряться расстоянием Хемминга $\chi(g, h)=n-\operatorname{im}\left(g h^{-1}\right), \operatorname{im}\left(g h^{-1}\right)$ - число неподвижных точек подстановки $g h^{-1}$. Нас будут интересовать два параметра:

- порядок $(w, r)$-примитивности, т. е. число

$$
\chi_{w, r}(g)=\min \left\{\chi(g, h) \mid h \in \mathrm{IG}_{W}, W \in W_{w, r}\right\} ;
$$

- порядок абсолютной примитивности, т. е. число

$$
\chi_{*}(g)=\min \left\{\chi_{w, r}(g) \mid w, r: w r=n, 1<r<n\right\} .
$$

Если $\chi_{w, r}(g)>0$, то подстановку $g$ будем называть $(w, r)$-примитивной. В противном случае - $(w, r)$-импримитивной. Отметим, что если число $n-1$ простое и $n>2$, то $\chi_{*}(g)=2$ для цикла длины $n-1$.

Нахождение порядка $(w, r)$-примитивности основано на том, что подстановки из множества $\mathrm{IG}_{(w, r)}$ имеют цикловую структуру, которая однозначно выделяет их среди всех остальных подстановок из симметрической группы $S(X)$. В работе описан порядок $(w, r)$-примитивности при чётном $n$ в крайних случаях $w=2$ и $r=2$. Приведены порядки $(w, r)$-примитивности для $s$-боксов некоторых известных криптосистем. Заметим, что задача нахождения порядка $(w, r)$-примитивности оказалась сложнее рассмотренной в [4].

Структура работы следующая. В разделе 2 приводятся основные обозначения и определения. В разделе 3 описывается цикловая структура подстановок из множества $\mathrm{IG}_{\mathrm{W}}$. В разделе 4 приведены оценки порядков $(w, r)$-примитивности. В разделе 5 найдены порядки $(w, r)$-примитивности для некоторых $s$-боксов известных криптосистем. 


\section{2. Основные обозначения и определения}

Пусть $W=\left\{W_{1}, \ldots, W_{r}\right\}$ - фиксированная система импримитивности с $r$ блоками импримитивности мощности $w$ каждый; $W_{w, r}$ - множество всех таких нетривиальных систем импримитивности с параметрами $w, r$. Далее максимальную импримитивную группу подстановок, сохраняющую систему импримитивности $W \in W_{w, r}$, будем обозначать $\mathrm{IG}_{W}=\left(S_{w} \prec S_{r}, W\right)$.

Будем придерживаться следующих обозначений:

- $\mathbb{N}$ - множество всех натуральных чисел, $\mathbb{N}_{0}=\mathbb{N} \cup\{0\} ;$

- $\operatorname{cs}(g)$ - цикловая структура подстановки $g \in S(X), \operatorname{cs}(g)=\left[1^{i_{1}}, 2^{i_{2}}, \ldots, n^{i_{n}}\right]$, если

$$
1 \cdot i_{1}+2 \cdot i_{2}+\ldots+n \cdot i_{n}=n
$$

и подстановка $g$ содержит $i_{j}$ циклов длины $j$;

- $M M \rrbracket-$ множество цикловых структур подстановок из множества $M$;

$-\bar{g} \in S_{r}$ - образ подстановки $g \in I G_{W}$ при естественном гомоморфизме $I G_{W} \rightarrow S_{r}$

- $\operatorname{In}(g)\left(\operatorname{In}^{\times}(g)\right)$ - множество всех независимых (см. [5]) циклов (неединичной длины) подстановки $g$;

- $\operatorname{In}_{q}(g)$ - множество всех независимых циклов подстановки $g$, имеющей точно $q$ циклов;

- $\operatorname{In}_{q}^{>1}(g)$ - множество всех независимых циклов неединичной длины подстановки $g$, имеющей точно $q$ циклов;

$-q_{g}=\left|\operatorname{In}_{q}^{>1}(g)\right| ;$

- $I_{q}(g)$ - множество всех независимых циклов длины $q$ подстановки $g$;

- $\alpha^{g}=\alpha g=g(\alpha)$ - образ элемента $\alpha \in X$ при действии на него подстановкой $g \in S(X)$

- $\operatorname{ord}(h)$ - порядок элемента $h \in S(X)$;

- $|h|$ - порядок цикла $h, \operatorname{ord}(h)=|h|$;

- $\alpha_{h}$ - элемент цикла $h \in \operatorname{In}(g)$, т. е.

$$
\left(\alpha_{h}\right)^{\langle h\rangle}=\left(\alpha_{h}, \alpha_{h}^{h}, . ., \alpha_{h}^{h^{\text {ord }(h)-1}}\right)=h ;
$$

- $h \circ h^{\prime}=\left(\alpha_{1}, \ldots, \alpha_{t_{1}}, \beta_{1}, \ldots, \beta_{t_{2}}\right)$ - объединение циклов, соответствующее двум циклам

$$
h=\left(\alpha_{1}, \ldots, \alpha_{t_{1}}\right), h^{\prime}=\left(\beta_{1}, \ldots, \beta_{t_{2}}\right),\left\{\alpha_{1}, \ldots, \alpha_{t_{1}}\right\} \cap\left\{\beta_{1}, \ldots, \beta_{t_{2}}\right\}=\varnothing ;
$$

$-\{h\}=\left\{\alpha_{1}, \ldots, \alpha_{t_{1}}\right\}$ для цикла $\left(\alpha_{1}, \ldots, \alpha_{t_{1}}\right)$ подстановки $h$. 


\section{3. Цикловая структура подстановок из множе- ства $\mathrm{IG}_{W}$}

Укажем ограничения, налагаемые на цикловую структуру подстановки $g$ включением $g \in \mathrm{IG}_{W}$.

Лемма 1. Если $g \in \mathrm{IG}_{W}, W \in W_{w, r}$, mo:

(1) Для каждого единичного ицкла $b \in \operatorname{In}(\bar{g})$ существует $p(b)$ таких попарно различных циклов $h_{b}^{(1)}, \ldots, h_{b}^{(p(b))} \in \operatorname{In}(g)$, что

$$
\sum_{j=1}^{p(b)} \operatorname{ord}\left(h_{b}^{(j)}\right)=w
$$

(2) Для каждого неединичного иикла $b \in \operatorname{In}(\bar{g})$ существует $p(b)$ таких попарно различных ииклов $h_{b}^{(1)}, \ldots, h_{b}^{(p(b))} \in \operatorname{In}(g)$, что

$$
\operatorname{ord}\left(h_{b}^{(i)}\right) \equiv 0(\bmod \operatorname{ord}(b)), \quad \sum_{i=1}^{p(b)} \operatorname{ord}\left(h_{b}^{(i)}\right)=w \cdot \operatorname{ord}(b) \text {. }
$$

Кроме того, $h_{t}^{(i)} \neq h_{t^{\prime}}^{\left(i^{\prime}\right)}$ для любъх $(i, t) \neq\left(i^{\prime}, t^{\prime}\right)$, где $t, t^{\prime} \in \operatorname{In}(\bar{g})$, $i \in\{1, \ldots, p(t)\}, i^{\prime} \in\left\{1, \ldots, p\left(t^{\prime}\right)\right\}$.

(3) Справедливы равенства

$$
\operatorname{In}(g)=\bigcup_{b \in \operatorname{In}(\bar{g})} \bigcup_{j=1}^{p(b)}\left\{h_{b}^{(j)}\right\}, \quad \sum_{b \in \operatorname{In}(\bar{g})} p(b)=|\operatorname{In}(g)| .
$$

Доказательство. Пусть $W=\left\{W_{1}, \ldots, W_{r}\right\}, d(\alpha)$ - длина орбиты группы $\langle g\rangle$, содержащей элемент $\alpha$.

1. Если $b$ - произвольный цикл длины 1 из $\operatorname{In}(\bar{g})$, то существует такой блок импримитивности $W_{t(b)}, t(b) \in\{1, \ldots, r\}$, что $W_{t(b)}^{g}=W_{t(b)}$.

Рассмотрим произвольный элемент $\alpha \in W_{t(b)}$. Тогда $\left(\alpha, \ldots, \alpha^{g^{d(\alpha)-1}}\right)$ - цикл длины $d(\alpha)$ со всеми элементами $\alpha^{g^{j}}$ из $W_{t(b)}, j \in\{1, \ldots, d(\alpha)-1\}$.

Так как $W_{t(b)}^{g}=W_{t(b)}$, то существуют такие элементы $\alpha_{1}, \ldots, \alpha_{p(b)}$, принадлежащие $W_{t(b)}$, что

$$
\bigcup_{c=1}^{p(b)} \bigcup_{j=0}^{d\left(\alpha_{c}\right)-1}\left\{\alpha_{c}^{g^{j}}\right\}=W_{t(b)}
$$

т. е. $\sum_{j=1}^{p(b)} d\left(\alpha_{j}\right)=w$. Значит, существуют $p(b)$ попарно различных циклов из $\operatorname{In}(g)$, сумма длин которых равна мощности $w$ блока импримитивности.

2. Рассмотрим произвольный цикл $b=\left(j_{0}, \ldots, j_{l-1}\right), b \in \operatorname{In}(\bar{g})$, подстановки $\bar{g}$ длины $l \geqslant 2$. Тогда $W_{j_{i}}^{g}=W_{j_{i+1(\bmod l)}}, i=0, \ldots, l-1$. Пусть $\alpha-$ произвольный элемент из $W_{j_{0}}$. Тогда $\alpha^{g^{t}} \in W_{j_{t}(\bmod l)}$ для $t=0, \ldots, d(\alpha)-1$. Следовательно, $d(\alpha) \equiv 0(\bmod l)$. 
Так как $W_{j_{i}}^{g}=W_{j_{i+1(\bmod |b|)}}, i=0, \ldots,|b|-1$, то существуют такие элементы $\alpha_{1}^{(b)}, \ldots, \alpha_{p(b)}^{(b)}$, принадлежащие $W_{j_{0}}$, что

$$
\begin{gathered}
d\left(\alpha_{j}^{(b)}\right) \equiv 0(\bmod |b|), j=1, \ldots, p(b), \\
\bigcup_{c=1}^{p(b)} \bigcup_{j=0}^{d\left(\alpha_{c}\right)-1}\left\{\alpha_{c}^{(b) g^{j}}\right\}=\bigcup_{i=1}^{|b|} W_{j_{i}},
\end{gathered}
$$

т. е. $\sum_{j=1}^{p(b)} d\left(\alpha_{j}^{(b)}\right)=|b| \cdot w$.

3. Положим $h_{b}^{(j)}=\left(\alpha_{j}^{(b)}\right)^{\langle g\rangle}, b \in \operatorname{In}(\bar{g}), j=1, \ldots, p(b)$. Из построения следует, что $\left(\alpha_{j}^{(b)}\right)^{\langle g\rangle} \neq\left(\alpha_{j^{\prime}}^{\left(b^{\prime}\right)}\right)^{\langle g\rangle}$ при $(j, b) \neq\left(j^{\prime}, b^{\prime}\right), b, b^{\prime} \in \operatorname{In}(\bar{g})$. Так как $W=\bigcup_{i=1}^{r} W_{i}$, то

$$
\operatorname{In}(g)=\bigcup_{b \in \operatorname{In}(\bar{g})} \bigcup_{j=1}^{p(b)}\left\{\left(\alpha_{j}^{(b)}\right)^{\langle g\rangle}\right\}, \quad \sum_{b \in \operatorname{In}(\bar{g})} p(b)=|\operatorname{In}(g)| .
$$

Перечислим цикловые структуры подстановок из множества $\mathrm{IG}_{(w, r)}=\bigcup_{W \in W_{w, r}} \mathrm{IG}_{W}$. Так как множество $\mathrm{IG}_{(w, r)}$ является объединением классов сопряжённых элементов группы $S(X)$, то цикловая структура элемента $g \in S(X)$ однозначно задаёт класс сопряжённых элементов, т. е. включение $g \in \mathrm{IG}_{(w, r)}$.

Утверждение 1. Тогда $и$ только тогда $g \in \mathrm{IG}_{W}$ для некоторого $W \in W_{w, r}$, когда существует такое разбиение $\widetilde{H}=\left(\widetilde{H_{1}}, \ldots, \widetilde{H_{d}}\right)$ множества $\operatorname{In}(g)$, что для каждого $i \in\{1, \ldots, d\}$ выполняется одно из двух условий:

(1) $\sum_{h \in \widetilde{H}_{i}} \operatorname{ord} h=w$.

(2) Существует число $l_{i} \in \mathbb{N}$, удовлетворяющее соотношениям:

(a) $\operatorname{ord} h \equiv 0\left(\bmod l_{i}\right)$ для всех $h \in \widetilde{H}_{i}$;

(b) $\sum_{h \in \widetilde{H}_{i}} \operatorname{ord} h=l_{i} \cdot w$.

Доказательство. Пусть существует система импримитивности $W=\left\{W_{1}, \ldots, W_{r}\right\} \in W_{w, r}$, для которой $W_{i}^{g}=W_{j(i)}$ для каждого $i \in\{1, \ldots, r\}$ и соответствующего $j(i) \in\{1, \ldots, r\}$. Пусть $\operatorname{In}(\bar{g})=\left\{b_{1}, \ldots, b_{p}\right\}$ - множество всех циклов подстановки $\bar{g} \in S_{r}$. Согласно лемме 1 для каждого цикла $b \in \operatorname{In}(\bar{g})$ существует такое множество циклов $H^{(b)}=\left\{h_{b}^{(1)}, \ldots, h_{b}^{(p(b))}\right\} \subseteq \operatorname{In}(g)$, что либо $\sum_{h \in H^{(b)}}|h|=w$, либо $|h| \equiv 0(\bmod |b|)$ для всех $h \in H^{(b)}$ и $\sum_{h \in H^{(b)}}|h|=|b| \cdot w$. Поэтому достаточно положить $\widetilde{H_{i}}=H^{\left(b_{i}\right)}, l_{i}=\left|b_{i}\right|, d=|\operatorname{In}(\bar{g})|=p, i=1, \ldots, p$.

Пусть теперь существует разбиение $\widetilde{H}=\left(\widetilde{H_{1}}, \ldots, \widetilde{H_{d}}\right)$ множества $\operatorname{In}(g)$, удовлетворяющее одному из двух условий формулировки данного утверждения. Зададим подстановку $\bar{g} \in S_{r}$ следующим алгоритмическим образом. Полагаем $X_{0}=\varnothing$.

Для $i=1, \ldots, d$ выполняем: 
(1) Если $i \in\{1, \ldots, d\}$ и $\sum_{h \in \widetilde{H}_{i}}|h|=w$, то выбираем произвольный элемент $j_{0}^{(i)} \in\{1, \ldots, r\} \backslash X_{i-1}$ и полагаем $\bar{g}: j_{0}^{(i)} \mapsto j_{0}^{(i)}, X_{i}=X_{i-1} \cup\left\{j_{0}^{(i)}\right\}$.

(2) Если $i \in\{1, \ldots, d\}$ и $|h| \equiv 0\left(\bmod l_{i}\right)$ для всех $h \in \widetilde{H}_{i}$ и $\sum_{h \in \widetilde{H}_{i}}|h|=l_{i} \cdot w$, то выбираем $l_{i}$ произвольных элементов $j_{0}^{(i)}, \ldots, j_{l_{i}-1}^{(i)}$ из множества $\{1, \ldots, r\} \backslash X_{i-1}$ и полагаем

$$
\bar{g}: j_{i} \rightarrow j_{i+1\left(\bmod l_{i}\right)}, X_{i}=X_{i-1} \cup\left\{j_{0}^{(i)}, \ldots, j_{l_{i}-1}^{(i)}\right\}
$$

Если $\sum_{h \in \widetilde{H}_{i}}|h|=w$, то полагаем $l_{i}=1$. Обозначим

$$
J_{i}=\left\{j_{0}^{(i)}, \ldots, j_{l_{i}-1}^{(i)}\right\}, l_{i}=\left|J_{i}\right|, i=1, \ldots, d
$$

Так как $\sum_{i=1}^{d} l_{i}=r$ и $J_{i}^{\bar{g}}=J_{i}$ для $i=1, \ldots, d$, то $\bar{g}$ - подстановка на множестве $\{1, \ldots, r\}$ и $J_{i}$ - орбита подстановки $\bar{g}$. Зададим теперь разбиение $\left(W_{1}, \ldots, W_{r}\right)$ множества $X$, для которого

$$
\left|W_{1}\right|=\ldots=\left|W_{r}\right|=w,
$$

$W_{i}^{g}=W_{j(i)}$ для каждого $i \in\{1, \ldots, r\}$ и соответствующего $j(i) \in\{1, \ldots, r\}$.

Для $i=1, \ldots, d$ выполняем:

(1) Если $\left|J_{i}\right|=1$, то полагаем $W_{j_{0}^{(i)}}=\left\{\alpha_{h} \mid h \in \widetilde{H}_{i}\right\}$.

(2) Если $\left|J_{i}\right|>1$, то полагаем

$$
\begin{aligned}
& W_{j_{0}^{(i)}}=\bigcup_{h \in \widetilde{H}_{i}} \bigcup_{t=0}^{|h| / l_{i}-1}\left\{\alpha_{h}^{h^{l_{i} \cdot t}}\right\} \\
& W_{j_{1}^{(i)}}=\bigcup_{h \in \widetilde{H}_{i}} \bigcup_{t=0}^{|h| / l_{i}-1}\left\{\alpha_{h}^{h^{l_{i} \cdot t+1}}\right\}, \ldots, W_{j_{l_{i}-1}}=\bigcup_{h \in \widetilde{H}_{i}}^{|h| / l_{i}-1} \bigcup_{t=0}\left\{\alpha_{h}^{h^{l_{i} \cdot t+l_{i}-1}}\right\} .
\end{aligned}
$$

Из построения следует, что для любого $h \in \widetilde{H}_{i}$ имеем: $W_{t}^{h}=W_{t}$ при $t \notin J_{i}$ и $W_{t}^{h}=W_{t^{\prime}}$ при $t, t^{\prime} \in J_{i}$. Следовательно, $g$ сохраняет разбиение $\left(W_{1}, \ldots, W_{r}\right)$, т. е. $W_{i}^{g}=W_{j(i)}$ для каждого $i \in\{1, \ldots, r\}$ и соответствующего $j(i) \in\{1, \ldots, r\}$. Значит, $g \in \mathrm{IG}_{W}$.

Очевидно, что $\chi_{w, r}(g)=\chi_{w, r}(g h)$ для всех $W \in W_{w, r}$ и всех $h \in \mathrm{IG}_{W}$. Значит, если существует хотя бы одна подстановка $g \in S(X)$, для которой $\chi_{w, r}(g)=i$, то таким же свойством обладает и множество подстановок $g \cdot\left(\bigcup_{W \in W_{w, r}} \mathrm{IG}_{W}\right)$. Из утверждения 1 следует, что $(w, r)$-примитивность подстановки $g \in S(X)$, как и порядок примитивности, определяется только её цикловой структурой, т. е. является функцией на классах сопряжённых элементов в группе $S(X)$. 


\section{4. Оценки порядка $(w, r)$-примитивности}

Для фиксированных $w, r \in \mathbb{N}, n=w \cdot r$, оценим снизу и сверху возможные значения расстояния $\chi_{w, r}(g)$.

Пусть $X \subseteq \mathbb{N}_{0}, X=\left\{x_{1}, \ldots, x_{n}\right\}$ - носитель мультимножества $\tilde{X}=\left[\left\{x_{1}^{\left(i_{1}\right)}, x_{2}^{\left(i_{2}\right)}, \ldots, x_{n}^{\left(i_{n}\right)}\right\}\right]$, в котором элемент $x_{j}$ присутствует $i_{j}$ раз,

$$
|\tilde{X}|=i_{1}+\ldots+i_{n}
$$

Величина $|\tilde{X}|$ - мощность мультимножества $\tilde{X}$. Если $i_{j} \in\{0,1\}, j=1, \ldots, n$, то

$$
\left\{x_{1} \cdot i_{1}, x_{2} \cdot i_{2}, \ldots, x_{n} \cdot i_{n}\right\} \backslash\{0\}=\left[\left\{x_{1}^{\left(i_{1}\right)}, x_{2}^{\left(i_{2}\right)}, \ldots, x_{n}^{\left(i_{n}\right)}\right\}\right] .
$$

Для мультимножества $B$ с носителем $X$ положим

$$
\sum_{b \in * B} b=x_{1} \cdot i_{1}+x_{2} \cdot i_{2}+\ldots+x_{n} \cdot i_{n} .
$$

Утверждение 2. Пусть $g \in S(X), r, w, q \in \mathbb{N}, n=r \cdot w, \operatorname{In}(g)=\left\{h_{1}, \ldots, h_{q}\right\}$. Тогда

$$
2 \leqslant \chi_{w, r}(g) \leqslant \min \left\{q_{g}+1, q, 2(r-1)\right\} .
$$

Доказательство. Зафиксируем произвольную систему импримитивности $W \in W_{w, r}$. Покажем сначала, что $2 \leqslant \chi_{w, r}(g)$.

Подстановка $s \in \mathrm{IG}_{W}$ может быть получена из подстановки $g$ умножением на некоторые транспозиции $v_{1}, \ldots, v_{t} \in S(X)$. Пусть $s^{\prime}=v_{1} \cdot \ldots \cdot v_{t}, s=g \cdot s^{\prime}$. Тогда $\chi\left(g, g \cdot v_{1}\right)=2$ для транспозиции $v_{1} \in S(X)$ и $\chi\left(g, g \cdot s^{\prime}\right) \geqslant 2$. Значит, $2 \leqslant \chi_{w, r}(g)$.

Покажем теперь справедливость оценки сверху

$$
\chi_{w, r}(g) \leqslant \min \left\{q_{g}+1, q, 2(r-1)\right\} .
$$

Пусть $\operatorname{In}(s)=\left\{h_{1}, \ldots, h_{q}\right\}$.

Из утверждения 1 следует, что любая полноцикловая подстановка принадлежит $\mathrm{IG}_{(w, r)}$. Определим полноцикловую подстановку $s \in S(X)$ равенством

$$
s=\left(\alpha_{h_{1}}, \alpha_{h_{1}}^{h_{1}}, \ldots, \alpha_{h_{1}}^{h_{1}^{\left|h_{1}\right|-1}}, \alpha_{h_{2}}, \alpha_{h_{2}}^{h_{2}}, \ldots, \alpha_{h_{2}}^{h_{2}^{\left|h_{2}\right|-1}}, \ldots, \alpha_{h_{q}}, \alpha_{h_{q}}^{h_{q}}, \ldots, \alpha_{h_{q}}^{h_{q}^{\left|h_{q}\right|-1}}\right) .
$$

Тогда $\chi(g, s)=q$. Следовательно, $\chi_{w, r}(g) \leqslant q$.

Пусть подстановка $g$ такова, что $q_{g}<q$. Положим $c=q_{g}$. Без ограничения общности допустим, что $\left|h_{i}\right|>1$ при $i=1, \ldots, q_{g},\left|h_{i}\right|=1$ при $i=q_{g}+1, \ldots, q$. Пусть также $\sum_{i=1}^{c}\left|h_{i}\right|=w t+j$, где $0 \leqslant j \leqslant w-1, t \geqslant 0$.

Если $t=0$, то $g \in \mathrm{IG}_{(w, r)}$ согласно утверждению 1 . При $t>0$ положим

$$
\begin{gathered}
s^{\prime}=\left(\alpha_{h_{1}}, \alpha_{h_{1}}^{h_{1}}, \ldots, \alpha_{h_{1}}^{h_{1}^{\left|h_{1}\right|-1}}, \alpha_{h_{2}}, \alpha_{h_{2}}^{h_{2}}, \ldots, \alpha_{h_{2}}^{h_{2}^{\left|h_{2}\right|-1}}, \ldots, \alpha_{c}, \ldots, \alpha_{c}^{h_{c}^{\left|h_{c}\right|-1}}\right)= \\
=\left(\beta_{1}, \ldots, \beta_{w t+j}\right), \\
s=\left(\beta_{1}, \ldots, \beta_{w t}\right)\left(\beta_{w t+1}, \ldots, \beta_{w t+j}\right) .
\end{gathered}
$$

Из утверждения 1 получаем, что $s \in \mathrm{IG}_{(w, r)}$. Так как $\chi(g, s)=q_{g}+1$, то $\chi_{w, r}(g) \leqslant q_{g}+1$. Зададим подстановку $s \in S(X)$ алгоритмическим образом.

Вход: $c_{1}=\ldots=c_{r}=0, j=1, i=1, H=\left\{h_{1}, \ldots, h_{q}\right\}, H^{\prime}=\varnothing$.

Пока $c_{j}<w, j \leqslant r, i<q$ выполняем: 
(1) Если $\left|h_{i}\right|<w-c_{j}$, то полагаем $H^{\prime} \leftarrow H^{\prime} \cup\left\{h_{i}\right\}, c_{j} \leftarrow c_{j}+\left|h_{i}\right|, i \leftarrow i+1$.

(2) Если $\left|h_{i}\right|=w-c_{j}$, то полагаем $H^{\prime} \leftarrow H^{\prime} \cup\left\{h_{i}\right\}, c_{j} \leftarrow w, j \leftarrow j+1, i \leftarrow i+1$.

(3) Если $\left|h_{i}\right|>w-c_{j}$, то при $h_{i}=\left(\alpha_{1}, \ldots, \alpha_{w-c_{i}}, \alpha_{w-c_{i}+1}, \ldots, \alpha_{\left|h_{i}\right|}\right)$ полагаем

$$
\begin{gathered}
l_{i}=\left|h_{i}\right|, H^{\prime} \leftarrow H^{\prime} \cup\left\{\left(\alpha_{1}, \ldots, \alpha_{w-c_{i}}\right)\right\}, \\
h_{i} \leftarrow\left(\alpha_{w-c_{i}+1}, \ldots, \alpha_{l_{i}}\right), c_{j} \leftarrow w, j \leftarrow j+1 .
\end{gathered}
$$

Въход: $\operatorname{In}(s)=H^{\prime}$.

Таким образом, получено множество $\operatorname{In}(s)$ независимых циклов подстановки $s$, задающих её однозначно. Из утверждения 1 следует, что существует такая система импримитивности, что $s \in \mathrm{IG}_{(w, r)}$. Из приведённого алгоритма следует, что

$$
\chi(g, s) \leqslant 2(r-1), \chi_{w, r}(g) \leqslant \min \left\{q_{g}+1, q, 2(r-1)\right\} .
$$

Будем говорить, что мультимножество $B^{(n)}$ с носителем из $\mathbb{N}_{0}$ является $(w, r)$ мультимножеством, если:

(1) $\left|B^{(n)}\right| \equiv 0(\bmod w),\left|B^{(n)}\right| \leqslant n$.

(2) Существует такое разбиение $\tilde{B}^{(n)}=\left\{B_{1}, \ldots, B_{c}\right\}$ мультимножества $B^{(n)}$, что для каждого $B_{j} \in \tilde{B}^{(n)}$ выполняется одно из условий:

(a) $\sum_{b \in B_{*} B_{j}} b=w$;

(b) существуют такое число $t_{j} \in \mathbb{N}$ и разбиение $\hat{B}_{j}=\left\{J_{1}, \ldots, J_{k}\right\}$ мультимножества $B_{j}$, что $\sum_{b \in \in_{*} B_{j}} b=t_{j} \cdot w$ и $\sum_{b \in \in_{*} J_{i}} b \equiv 0\left(\bmod t_{i}\right)$ для $i=1, \ldots, k$.

Назовём мультимножество $P$-свободнъм, если не существует собственного мультимножества $B^{(n)} \subset P$, являющегося $(w, r)$-мультимножеством. Подстановку $g \in S(X)$ с цикловой структурой $\left[1^{i_{1}}, 2^{i_{2}}, \ldots, n^{i_{n}}\right]$, у которой мультимножество $\left[\left\{1^{\left(i_{1}\right)}, 2^{\left(i_{2}\right)}, \ldots, n^{\left(i_{n}\right)}\right\}\right]$ является $(w, r)$-свободным, также будем называть $(w, r)$ свободной.

Пусть подстановка $g \in S(X)$ имеет цикловую структуру $\left[1^{i_{1}}, 2^{i_{2}}, \ldots, n^{i_{n}}\right]$ и $B^{(n)}=\left[\left\{1^{\left(j_{1}\right)}, 2^{\left(j_{2}\right)}, \ldots, n^{\left(j_{n}\right)}\right\}\right]$ является $(w, r)$-мультимножеством из $\left[\left\{1^{\left(i_{1}\right)}, 2^{\left(i_{2}\right)}, \ldots, n^{\left(i_{n}\right)}\right\}\right]$ наибольшей мощности, при этом $j_{t} \leqslant i_{t}$ для $t=1, \ldots, n$.

Обозначим через $\operatorname{In}\left(g, B^{(n)}\right)$ такое произвольное подмножество множества циклов $\operatorname{In}(g)$, что мультимножество, состоящее из всех длин циклов из $\operatorname{In}\left(g, B^{(n)}\right)$, совпадает с $B^{(n)}$.

Также положим

$$
X_{g, B^{(n)}}=\bigcup_{h \in \operatorname{In}\left(g, B^{(n)}\right)}\{h\}, \quad X_{g, B^{(n)}}^{\prime}=\bigcup_{h \in \operatorname{In}(g) \backslash\left(\operatorname{In}\left(g, B^{(n)}\right)\right)}\{h\} .
$$

Тогда подстановка $g$ представима в виде $g=g^{\prime} \tilde{g}$, где $g^{\prime} \in S\left(X_{g, B^{(n)}}\right)$, $\tilde{g} \in S\left(X_{g, B^{(n)}}^{\prime}\right)$. При этом $\chi_{w, r}\left(g^{\prime}\right)=0$ и $\chi_{w, r}(g)=\chi_{w, r}(\tilde{g})$. Кроме того, мультимножество $\left[\left\{1^{\left(i_{1}\right)}, 2^{\left(i_{2}\right)}, \ldots, n^{\left(i_{n}\right)}\right\}\right] \backslash B^{(n)}$ и подстановка $\tilde{g}$ являются $(w, r)$-свободными. 
Таким образом, достаточно найти расстояние до множества $\mathrm{IG}_{(w, r)}$ только для $(w, r)$ свободных подстановок.

При заданных $r, w \in \mathbb{N}, n=r \cdot w$, назовём подстановку $g \in S(X)$ максимально $(w, r)$-примитивной, если она максимально удалена от множества $\mathrm{IG}_{(w, r)}$ в метрике Хемминга. Ясно, что необходимым условием максимальной $(w, r)$-примитивности подстановки $g$ является её $(w, r)$-свободность.

Максимально $(w, r)$-примитивные подстановки являются аналогом бентфункций. При этом аналогом множества всех аффинных функций служит множество подстановок $\mathrm{IG}_{(w, r)}$. Нахождение максимально $(w, r)$-примитивных подстановок также является довольно сложной задачей.

Пусть $g$ - произвольная подстановка из $S(X)$. Произвольное множество $P \subseteq \operatorname{In}(g)$ будем называть 2-правильным, если существует такой цикл $y \in P$, что НОД $\left(\sum_{x \in P \backslash\{y\}}\right.$ ord $\left.x, \operatorname{ord} y\right)>1$ и $\sum_{x \in P}$ ord $x$ - чётное число.

Для подстановки $g \in S(X)$ обозначим:

- $I_{2 i-1}^{\prime}(g)$ - произвольное подмножество множества $I_{2 i-1}(g)$ наибольшей чётной мощности, где $I_{2 i-1}(g) \neq \varnothing$ и $i=1, \ldots,\lfloor n / 2\rfloor$;

$-H^{\prime}(g)=\operatorname{In}(g) \backslash\left(\left(\bigcup_{i=1}^{\lfloor n / 2\rfloor} I_{2 i}(g)\right) \cup\left(\bigcup_{i=1}^{\lfloor n / 2\rfloor} I_{2 i-1}^{\prime}(g)\right)\right), q(g)=\left|H^{\prime}(g)\right| ;$

- $t(g)$ - наибольшее число попарно не пересекающихся 2-правильных подмножеств $P_{1}(g), \ldots, P_{t(g)}(g)$ множества $H^{\prime}(g)$.

Утверждение 3. Если $n=r \cdot 2 u g \in S(X)$, mo

$$
\chi_{2, r}(g)=q(g)-t(g) .
$$

Доказательство. Положим

$$
\begin{gathered}
H^{\prime \prime}=\left(\left(\bigcup_{i=1}^{\lfloor n / 2\rfloor} I_{2 i}(g)\right) \cup\left(\bigcup_{i=1}^{\lfloor n / 2\rfloor} I_{2 i-1}^{\prime}(g)\right)\right), \\
s^{\prime}=\prod_{b \in H^{\prime \prime}} b, s^{\prime \prime}=\prod_{b \in H^{\prime}(g)} b .
\end{gathered}
$$

Согласно утверждению 1 имеем $\chi_{2, r}\left(s^{\prime}\right)=0$ и $\chi_{2, r}(g)=\chi_{2, r}\left(s^{\prime} s^{\prime \prime}\right)=\chi_{2, r}\left(s^{\prime \prime}\right)$. Кроме того, $\operatorname{In}\left(s^{\prime \prime}\right)=H^{\prime}(g)$. Согласно заданию множество $H^{\prime}(g)$ содержит только нечётные циклы разной длины.

Пусть

$$
\begin{gathered}
H^{\prime}(g)=\left\{b_{1}, \ldots, b_{q^{\prime}(g)}\right\} \cup \bigcup_{c=1}^{t(g)} P_{c}(g), \quad\left\{b_{1}, \ldots, b_{q^{\prime}(g)}\right\} \cap \bigcup_{c=1}^{t(g)} P_{c}(g)=\varnothing, \\
P_{c}(g)=\left\{b_{i_{1}}^{(c)}, \ldots, b_{i_{j_{c}}}^{(c)}\right\}, \quad P_{c}(g) \subset H^{\prime}(g), \\
\text { НОД }\left(\left|b_{i_{2}}^{(c)}\right|+\ldots+\left|b_{i_{j_{c}}}^{(c)}\right|, \quad\left|b_{i_{1}}^{(c)}\right|\right)>1, c=1, \ldots, t(g), \\
H^{\prime \prime}(g)=\left\{b_{1}, \ldots, b_{q^{\prime}(g)}\right\}=H^{\prime}(g) \backslash \bigcup_{c=1}^{t(g)} P_{c}(g) .
\end{gathered}
$$


Так как $t(g)$ - наибольшее число не пересекающихся 2-правильных подмножеств $P_{1}(g), \ldots, P_{t(g)}(g)$ множества $H^{\prime}(g)$, то мультимножество $H^{\prime \prime}(g)$ не содержит 2 -правильных подмножеств. Кроме того, $q(g)$ - чётное число.

Положим

$$
h=\left(b_{1}, \ldots, b_{q^{\prime}(g)}\right) \cdot \prod_{c=1}^{t(g)} b_{i_{1}}^{(c)} \cdot\left(b_{i_{2}}^{(c)}, \ldots, b_{i_{j_{c}}}^{(c)}\right) .
$$

Так как НОД $\left(\left|b_{i_{2}}^{(c)}\right|+\ldots+\left|b_{i_{j_{c}}}^{(c)}\right|,\left|b_{i_{1}}^{(c)}\right|\right)>1$ и $\left|b_{i_{1}}^{(c)}\right|+\ldots+\left|b_{i_{j_{c}}}^{(c)}\right|$ чётно, $c=1, \ldots, t(g)$, то из утверждения 1 следует, что $h \in \mathrm{IG}_{(2, r)}$. Из задания подстановки $h$ получаем

$$
\chi\left(h, s^{\prime \prime}\right)=q^{\prime}(g)+\sum_{c=1}^{t(g)}\left(j_{c}-1\right)=q(g)-t(g) .
$$

Предположим, что существует подстановка $h^{\prime} \in \mathrm{IG}_{(2, r)}$, для которой

$$
\chi\left(h^{\prime}, s^{\prime \prime}\right)<q(g)-t(g) .
$$

Пусть

$-f_{j}^{(1)}: S(X) \rightarrow S(X)$ - разбиение цикла длины $l$ на два цикла меньшей длины $j, l-j$, где $j \in\{1, \ldots, l-1\}$;

- $f^{(2)}: S(X) \rightarrow S(X)$ - объединение двух независимых циклов в один цикл, т. е. объединением циклов $\left(\alpha_{1}, \ldots, \alpha_{l_{1}}\right),\left(\beta_{1}, \ldots, \beta_{l_{2}}\right)$ является цикл $\left(\alpha_{1}, \ldots, \alpha_{l_{1}}, \beta_{1}, \ldots, \beta_{l_{2}}\right)$;

- $g \longrightarrow{\left(t_{1}, t_{2}\right)} s-$ подстановка $s \in S(X)$ получена посредством $t_{1}$ элементарных операций $f_{j}^{(1)}$ и $t_{2}$ элементарных операций $f^{(2)}$ из подстановки $g$.

Таким образом,

$$
\begin{aligned}
& f_{j}^{(1)}:\left(\alpha_{1}, \ldots, \alpha_{j}, \alpha_{j+1}, \ldots, \alpha_{l}\right) \rightarrow\left(\alpha_{1}, \ldots, \alpha_{j}\right)\left(\alpha_{j+1}, \ldots, \alpha_{l}\right), \\
& f^{(2)}:\left(\alpha_{1}, \ldots, \alpha_{j}\right)\left(\alpha_{j+1}, \ldots, \alpha_{l}\right) \rightarrow\left(\alpha_{1}, \ldots, \alpha_{j}, \alpha_{j+1}, \ldots, \alpha_{l}\right) .
\end{aligned}
$$

Пусть $s^{\prime \prime} \longrightarrow{\left(t_{1}, t_{2}\right)}^{\prime}, t_{1}>0$ и $t_{2} \geqslant 0, t_{1}+t_{2}<q(g)-t(g)$. Если к каждому из элементов множества $H^{\prime}(g)$ при построении $h^{\prime}$ из $s^{\prime \prime}$ применена одна из операций $f_{j}^{(1)}, f^{(2)}$, то в этом случае заведомо $\chi\left(h^{\prime}, s^{\prime \prime}\right) \geqslant q(g)$. Следовательно, существует по крайней мере $t(g)+1$ циклов, к которым не применена ни одна из операций $f_{j}^{(1)}, f^{(2)}$.

Пусть $b^{\prime}$ - произвольный цикл из $H^{\prime}(g)$, к которому не применена ни одна из операций $f_{j}^{(1)}, f^{(2)}$. Из утверждения 1 следует, что должен существовать такой цикл $\tilde{b}^{\prime}$, что $\left|\tilde{b}^{\prime}\right|+\left|b^{\prime}\right|$ - чётное число и НОД $\left(\left|\tilde{b}^{\prime}\right|,\left|b^{\prime}\right|\right)>1$. Такой цикл $\tilde{b}^{\prime}$ может быть получен либо объединением некоторых циклов из $H^{\prime}(g)$, либо разбиением некоторых циклов из $H^{\prime}(g)$ и затем объединением получившихся циклов с некоторыми циклами из $H^{\prime}(g)$. Если цикл $\tilde{b}^{\prime}$ получен объединением некоторых циклов из $H^{\prime}(g)$, то цикл $b^{\prime}$ принадлежит некоторому 2-правильному подмножеству множества $H^{\prime}(g)$. Число $t(g)$ определяет наибольшее число таких непересекающихся подмножеств множества $H^{\prime}(g)$. 
Пусть $H^{\prime \prime \prime}(g)$ - множество всех циклов, полученных после разбиения некоторых циклов из $H^{\prime}(g)$ с целью дальнейшего объединения части из них для получения подстановки $h^{\prime}$. Пусть также $\widetilde{H^{\prime}}(g)$ - множество всех циклов, к которым не применена ни одна из операций $f_{j}^{(1)}, f^{(2)}$. Для $x \in H^{\prime}(g)$ положим $x \longrightarrow f_{f^{(1)}} x^{\prime}$, если $x^{\prime}=x$ или $x^{\prime}$ является частью подряд идущих элементов цикла $x$, полученной при разбиении цикла $x$ применением операции $f_{j}^{(1)}$.

Пусть $s_{X}$ - ограничение подстановки $s$ на множество $X=\left\{x_{1}, \ldots, x_{m}\right\}, x_{1}<$ $x_{2}<\ldots<x_{m}$, т. е.

$$
s_{X}=\left(\begin{array}{ccc}
x_{1} & \ldots & x_{m} \\
x_{1}^{s} & \ldots & x_{m}^{s}
\end{array}\right) .
$$

Для $b^{\prime} \in \widetilde{H^{\prime}}(g), x \in H^{\prime}(g)$ через $U\left(b^{\prime}, \tilde{b}^{\prime}\right)$ обозначим множество всех циклов из $H^{\prime \prime \prime}(g) \backslash \widetilde{H^{\prime}}(g)$, объединением которых является цикл $\tilde{b}^{\prime}$, и положим

$$
u\left(\left(b^{\prime}, \tilde{b}^{\prime}\right), x\right)=\left\{\begin{array}{l}
1, \quad \text { если } \exists x^{\prime} \in U\left(b^{\prime}, \tilde{b}^{\prime}\right): x \longrightarrow_{f^{(1)}} x^{\prime}, \\
0 \quad \text { иначе. }
\end{array}\right.
$$

Тогда

$$
\chi\left(s^{\prime \prime}\left\{\left(b^{\prime}, \tilde{b}^{\prime}\right)\right\},\left(b^{\prime}, \tilde{b}^{\prime}\right)\right)=\sum_{x \in H^{\prime}(g)} u\left(\left(b^{\prime}, \tilde{b}^{\prime}\right), x\right) .
$$

Пусть $\widetilde{H^{\prime}}{ }_{1}(g)$ - множество всех циклов $b^{\prime}$ из $\widetilde{H^{\prime}}(g)$, для которых

$$
U\left(b^{\prime}, \tilde{b}^{\prime}\right) \cap H^{\prime}(g)=U\left(b^{\prime}, \tilde{b}^{\prime}\right),
$$

т. е. каждый цикл $x \in U\left(b^{\prime}, \tilde{b}^{\prime}\right)$ являлся независимым циклом подстановки $s^{\prime \prime}$ и он не был разбит на циклы операцией $f_{j}^{(1)}, \widetilde{H^{\prime}}{ }_{2}(g)=\widetilde{H^{\prime}}(g) \backslash \widetilde{H^{\prime}}{ }_{1}(g)$. Положим

$$
\begin{gathered}
H_{1}^{\prime}(g)=\left(\bigcup_{b^{\prime} \in \widetilde{H^{\prime}{ }_{1}}(g)} U\left(b^{\prime}, \tilde{b}^{\prime}\right)\right) \cup \widetilde{{H^{\prime}}_{1}}(g), \\
H_{2}^{\prime}(g)=H^{\prime}(g) \backslash\left({H^{\prime}}_{1}(g) \cup \widetilde{{H^{\prime}}_{2}}(g)\right) .
\end{gathered}
$$

Выполняется равенство

$$
\begin{gathered}
\chi\left(s^{\prime \prime}, h^{\prime}\right)=\sum_{b^{\prime} \in \widetilde{H}^{\prime}(g)} \chi\left(s^{\prime \prime}\left\{\left(b^{\prime}, \tilde{b}^{\prime}\right)\right\},\left(b^{\prime}, \tilde{b}^{\prime}\right)\right)= \\
=\sum_{b^{\prime} \in \widetilde{H^{\prime}} 1(g)} \chi\left(s^{\prime \prime}\left\{\left(b^{\prime}, \tilde{b}^{\prime}\right)\right\},\left(b^{\prime}, \tilde{b}^{\prime}\right)\right)+\sum_{b^{\prime} \in \widetilde{H^{\prime}}{ }_{2}(g)} \chi\left(s^{\prime \prime}\left\{\left(b^{\prime}, \tilde{b}^{\prime}\right)\right\},\left(b^{\prime}, \tilde{b}^{\prime}\right)\right) .
\end{gathered}
$$

Первую сумму в правой части равенства (3) можно оценить снизу:

$$
\sum_{b^{\prime} \in \widetilde{H^{\prime}} 1(g)} \chi\left(s^{\prime \prime}\left\{\left(b^{\prime}, \tilde{b}^{\prime}\right)\right\},\left(b^{\prime}, \tilde{b}^{\prime}\right)\right)=\left|U\left(b^{\prime}, \tilde{b}^{\prime}\right)\right| \geqslant\left|H^{\prime}{ }_{1}(g)\right|-t(g) .
$$


Вторая сумма в правой части равенства (3) тоже оценивается снизу:

$$
\begin{gathered}
\sum_{b^{\prime} \in \widetilde{H^{\prime}}{ }_{2}(g)} \chi\left(s^{\prime \prime}\left\{\left(b^{\prime}, \tilde{b}^{\prime}\right)\right\},\left(b^{\prime}, \tilde{b}^{\prime}\right)\right)=\sum_{b^{\prime} \in \widetilde{H^{\prime}{ }_{2}}(g)} \sum_{x \in H^{\prime}{ }_{2}(g)} u\left(\left(b^{\prime}, \tilde{b}^{\prime}\right), x\right)= \\
=\sum_{x \in H^{\prime}{ }_{2}(g)} \sum_{b_{b^{\prime} \in \widetilde{H^{\prime}}{ }_{2}(g)} u\left(\left(b^{\prime}, \tilde{b}^{\prime}\right), x\right) \geqslant\left|\widetilde{H^{\prime}}{ }_{2}(g)\right|+\left|H^{\prime}{ }_{2}(g)\right| .}
\end{gathered}
$$

Действительно, каждый цикл $x \in H_{2}^{\prime}(g)$ принадлежит множеству $U\left(b^{\prime}, \tilde{b}^{\prime}\right)$ для некоторого $b^{\prime} \in \widetilde{{H^{\prime}}_{2}}(g)$, и для каждого $b^{\prime} \in \widetilde{{H^{\prime}}_{2}}(g)$ найдётся хотя бы один $x \in$ $U\left(b^{\prime}, \tilde{b}^{\prime}\right)$, для которого $u\left(\left(b^{\prime}, \tilde{b}^{\prime}\right), x\right)=u((b, \tilde{b}), x)$ при некотором $b^{\prime} \in \widetilde{H^{\prime}}{ }_{2}(g) \backslash\left\{b^{\prime}\right\}$.

Подставляя в равенство (3) оценки (4), (5) и используя равенство

$$
\left|H^{\prime}{ }_{1}(g)\right|+\left|\widetilde{H^{\prime}}{ }_{2}(g)\right|+\left|H^{\prime}{ }_{2}(g)\right|=\left|H^{\prime}(g)\right|=q(g),
$$

получаем

$$
\chi\left(s^{\prime \prime}, h^{\prime}\right) \geqslant\left|\widetilde{H^{\prime}}{ }_{2}(g)\right|+\left|H_{2}^{\prime}(g)\right|+\left|H^{\prime}{ }_{1}(g)\right|-t(g) \geqslant q(g)-t(g) .
$$

Следовательно получили противоречие с неравенством (2). Таким образом, из равенства (1) следует, что

$$
\chi_{2, r}(g)=q(g)-t(g)
$$

Отметим, что в доказательстве утверждения 3 для подстановки $g \in S(X)$ находится подстановка $h \in \mathrm{IG}_{(2, r)}$, лежащая на минимальном расстоянии $\chi(g, h)=q(g)-t(g)$ от подстановки $g$ среди всех $(w, r)$-импримитивных подстановок. Кроме того, множество $H^{\prime}(g)$ является $(w, r)$-свободным. Таким образом, если в условиях утверждения 3 выполняется включение $H^{\prime}(g) \subset \operatorname{In}(g)$, то существует такое подмножество $H \subseteq \operatorname{In}(g)$ наибольшей мощности, что $\prod_{h \in H} h \in \mathrm{IG}_{(2, r)}$. В этом случае подстановка $g$ представима в виде произведения подстановок $s^{\prime} s^{\prime \prime}$, где $s^{\prime}=\prod_{h \in H} h$, a $s^{\prime \prime}$ можно рассматривать как подстановку из $S\left(X^{\prime}\right)$, где $X^{\prime}=H^{\prime}(g) \backslash H$ и $\left|X^{\prime}\right|<|X|$.

В утверждении 3 указано расстояние от произвольной подстановки $g \in S(X)$ до множества $\mathrm{IG}_{(2, r)}$. В случае произвольных $(w, r)$ нахождение расстояния до множества $\mathrm{IG}_{(w, r)}$ является сложной задачей. Как и в случае бент-функций, расстояние до $\mathrm{IG}_{(w, r)}$ можно найти (или получить для него оценку сверху) лишь для некоторых классов подстановок.

Пусть $n$ чётно. Множество нечётных чисел $Y_{n} \subseteq\{1, \ldots, n\}$ назовём 2-максимальным, если оно чётной и максимальной мощности среди множеств, удовлетворяющих условию $\sum_{y \in Y_{n}} y \leqslant n$ для каждого $x \in Y_{n}$, и справедливо равенство НОД $\left(x, \sum_{y \in Y} y\right)=1$ для каждого подмножества $Y \subseteq Y_{n} \backslash\{x\}$.

Из утверждения 2 следует, что $\chi_{w, r}(g) \leqslant|\operatorname{In}(g)|$ для произвольной подстановки $g \in S(X)$. Укажем семейство подстановок, для которых достигается равенство. Оказывается, что достижимость данного равенства существенно зависит от «арифметических свойств» числа $n$, а именно, от существования 2-максимального множества $Y_{n} \subseteq\{1, \ldots, n\}$ со свойством $\sum_{y \in Y_{n}} y=n$.

Следствие 1. Пусть $r \in \mathbb{N}, n=2 r, g-$ произвольная подстановка из $S(X)$ с цикловой структурой $\left[1^{i_{1}}, 2^{i_{2}}, \ldots, n^{i_{n}}\right]$. Тогда и только тогда $\chi_{w, r}(g)=|\operatorname{In}(g)|$, когда $\left[\left\{1^{\left(i_{1}\right)}, 2^{\left(i_{2}\right)}, \ldots, n^{\left(i_{n}\right)}\right\}\right]$ - 2-максимальное множество. 
Доказательство. следует из утверждения 3.

Пусть $v\left(Q_{n}\right)$ - наибольшее число попарно не пересекающихся 2-правильных подмножеств $Q_{1}^{\prime}, \ldots, Q_{t\left(P_{n}\right)}^{\prime}$ множества $Q_{n}$.

Множество нечётных чисел $P_{n} \subseteq\{1, \ldots, n\}$ назовём 2-квазипримитивным, если оно удовлетворяет одновременно следующим условиям:

(1) $\sum_{y \in P_{n}} y \leqslant n$ и НОД $(x, y)=1$ для любых $x, y \in P_{n}, x \neq y$.

(2) Величина $\left|P_{n}\right|-v\left(P_{n}\right)$ является наибольшей по всем множествам, удовлетворяющим условию 1.

Положим $\omega(n)=\left|Y_{n}\right|$ для некоторого 2-максимального множества $Y_{n}$ и $\bar{\omega}(n)=\left|P_{n}\right|-v\left(P_{n}\right)$ для некоторого 2-квазипримитивного множества $P_{n}$.

Утверждение 4. Пусть $n=2 r, g \in S(X), \operatorname{cs}(g)=\left[1^{i_{1}}, 2^{i_{2}}, \ldots, n^{i_{n}}\right] u$ $\left[\left\{1^{\left(i_{1}\right)}, 2^{\left(i_{2}\right)}, \ldots, n^{\left(i_{n}\right)}\right\}\right]$ - 2-квазипримитивное множество. Тогда:

(1) $\chi_{w, r}(g)=\bar{\omega}(n)$.

(2) $\omega(n) \leqslant \chi_{w, r}(g)$.

Доказательство. П. 1 следует из утверждения 3. Для доказательства п. 2 используем обозначения утверждения 2 . Оценка $\omega(n) \leqslant \chi_{w, r}(g)$ следует из того, что согласно следствию 1 существует подстановка $h \in S(X), \operatorname{cs}(h)=\left[1^{i_{1}}, 2^{i_{2}}, \ldots, n^{i_{n}}\right]$, у которой множество $H^{\prime}(g)$ является 2-максимальным. Значит, $\chi_{2, r}(g)=\omega(n)$.

Следствие 1 и приводимые далее примеры показывают, что для некоторых $n$ равенство $\omega(n)=\chi_{w, r}(g)$ достижимо. Опишем расстояние $\chi_{w, r}(g)$ для чётного $n=2 r$. Используя утверждения 2 и 3 , несложно показать, что:

(1) Если $n=2 w, g \in S(X)$ и $\operatorname{cs}(g) \notin\left[\left[\operatorname{IG}_{(w, 2)}\right]\right]$, то $\chi_{w, 2}(g)=2$.

(2) Если $n=4 w$, то $\left\{\chi_{w, 4}(g) \mid g \in S(X)\right\}=\{2,3,4\}$.

Пример. Пусть $n=16$.

(1) Пусть $(w, r) \in\{(4,4),(8,2)\}$. Все подстановки, не принадлежащие множеству $\mathrm{IG}_{(w, r)}$, лежат от него на расстоянии 2 .

(2) Пусть $w=2, r=8$. На расстоянии 2 от $\mathrm{IG}_{(2,8)}$ лежат все подстановки, у которых цикловая структура не принадлежит $\left[\left[\mathrm{IG}_{(2,8)}\right]\right] \cup\left[1^{1}, 3^{1}, 5^{1}, 7^{1}\right]$. Наибольшим является расстояние 3 . Соответствующие подстановки имеют цикловую структуру $\left[1^{1}, 3^{1}, 5^{1}, 7^{1}\right]$.

Пусть $\mathrm{IG}_{*}$ - множество всех импримитивных подгрупп группы $S(X)$. Для произвольной подстановки $g \in S(X)$ обозначим

$$
\chi_{\mathrm{impr}}(g)=\min \left\{\chi(g, G) \mid G \leqslant \mathrm{IG}_{*}\right\} .
$$

Утверждение 5. Пусть $n, q \in \mathbb{N}, g \in \operatorname{In}_{q}(X), r$ - наименъший собственный простой делитель числа $n$. Тогда

$$
\chi_{\mathrm{impr}}(g) \leqslant \min \{q, 2(r-1)\} .
$$


Доказательство следует из утверждения 2, так как существует импримитивная группа $\mathrm{IG}_{W}=\left(S_{w} 2 S_{r}, W\right)$ с мощностью блоков импримитивности, равной $w=n / r$.

Следствие 2. Пусть $n-$ произвольное чётное число. Тогда $\chi_{\mathrm{impr}}(g)=2$ для любой подстановки $g \in S(X) \backslash\left(\bigcup_{G \in \mathrm{IG}_{*}} G\right)$.

\section{5. Расстояния $\chi_{w, r}$ до множеств $\mathrm{IG}_{(w, r)}$ для некоторых классов преобразований}

Приведём расстояния $\chi_{w, r}$ для некоторых $s$-боксов известных криптосистем AES, ARIA, MISTY1, Camellia, FOX (см., например, [6]), Whirlpool [7], SMS4 [8].

- Шифрсистема AES. Цикловая структура s-бокса есть $\left[2^{1}, 27^{1}, 59^{1}, 81^{1}, 87^{1}\right]$. Так как НОД $(87,81)=3$ и $87+81 \equiv 0(\bmod 8)$, то из утверждения 3 следует, что

$$
\begin{gathered}
\chi_{2,128}(s)=\chi_{4,64}(s)=\chi_{8,32}(s)=\chi_{128,2}(s)=2, \\
\chi_{16,16}(s)=\chi_{32,8}(s)=\chi_{64,4}(s)=4 .
\end{gathered}
$$

- Шифрсистема SMS4. Цикловая структура s-бокса есть $\left[1^{1}, 3^{1}, 6^{1}, 9^{1}, 24^{1}, 35^{1}, 56^{1}, 122^{1}\right]$. Так как НОД $(3,9)=3$ и $3+9 \equiv 0(\bmod 4)$, то из утверждения 3 следует, что

$$
\begin{gathered}
\chi_{2,128}(s)=\chi_{4,64}(s)=\chi_{32,8}(s)=\chi_{128,2}(s)=\chi_{16,16}(s)=2, \\
\chi_{8,32}(s)=\chi_{64,4}(s)=4 .
\end{gathered}
$$

- Шифрсистема ARIA. Цикловая структура $s$-бокса есть $\left[2^{1}, 3^{1}, 9^{1}, 21^{1}, 36^{1}, 64^{1}, 121^{1}\right]$. Так как НОД $(3,9)=3$ и $3+9 \equiv 0(\bmod 4)$, то из утверждения 3 следует, что

$$
\begin{gathered}
\chi_{2,128}(s)=\chi_{4,64}(s)=\chi_{32,8}(s)=\chi_{128,2}(s)=2, \\
\chi_{8,32}(s)=\chi_{16,16}(s)=\chi_{64,4}(s)=3 .
\end{gathered}
$$

- Kpиптосистема Whirlpool. Цикловая структура s-бокса есть $\left[2^{1}, 3^{2}, 6^{1}, 8^{3}, 12^{2}, 20^{1}, 82^{1}, 92^{1}\right]$. Имеем

$$
\begin{gathered}
\chi_{2,128}(s)=\chi_{4,64}(s)=\chi_{128,2}(s)= \\
=\chi_{8,32}(s)=\chi_{16,16}(s)=\chi_{32,8}(s)=\chi_{64,4}(s)=0 .
\end{gathered}
$$

- Шифрсистема MISTY1. Используются две подстановки $s_{1} \in S\left(V_{7}\right)$ и $s_{2} \in S\left(V_{9}\right)$. Цикловая структура подстановки $s_{1}$ есть $\left[1^{1}, 2^{1}, 7^{1}, 9^{1}, 17^{1}, 20^{1}, 26^{1}, 46^{1}\right]$. Имеем

$$
\begin{gathered}
\chi_{2,64}\left(s_{1}\right)=\chi_{4,32}\left(s_{1}\right)=\chi_{8,16}\left(s_{1}\right)=4, \\
\chi_{32,4}\left(s_{1}\right)=2, \chi_{64,2}\left(s_{1}\right)=0 .
\end{gathered}
$$


Цикловая структура подстановки $s_{2}$ есть $\left[6^{1}, 48^{1}, 86^{1}, 110^{1}, 128^{1}, 134^{1}\right]$. Так как длины всех циклов делятся на два, то

$$
\begin{aligned}
\chi_{2,256}\left(s_{2}\right)=\chi_{4,128}\left(s_{2}\right)=\chi_{128,4}\left(s_{2}\right)=\chi_{8,64}\left(s_{2}\right)= \\
=\chi_{16,32}\left(s_{2}\right)=\chi_{32,16}\left(s_{2}\right)=\chi_{64,8}\left(s_{2}\right)=\chi_{256,2}\left(s_{2}\right)=0 .
\end{aligned}
$$

- Шифрсистема Camellia. Рассмотрим два $s$-бокса $s_{1}, s_{2} \in S\left(V_{8}\right)$. Цикловая структура подстановки $s_{1}$ есть $\left[249^{1}, 5^{1}, 2^{1}\right]$. Отсюда

$$
\begin{gathered}
\chi_{2,128}\left(s_{1}\right)=\chi_{4,64}\left(s_{1}\right)=\chi_{128,2}\left(s_{1}\right)= \\
=\chi_{8,32}\left(s_{1}\right)=\chi_{16,16}\left(s_{1}\right)=\chi_{32,8}\left(s_{1}\right)=\chi_{64,4}\left(s_{1}\right)=2 .
\end{gathered}
$$

Цикловая структура подстановки $s_{2}$ есть $\left[13^{1}, 10^{1}, 51^{1}, 71^{1}, 111^{1}\right]$. Учитывая, что НОД $(51,111)=3,51+111 \equiv 0(\bmod 2)$, имеем

$$
\begin{gathered}
\chi_{2,128}\left(s_{2}\right)=\chi_{64,4}\left(s_{2}\right)=\chi_{128,2}\left(s_{2}\right)=2, \\
\chi_{4,64}\left(s_{2}\right)=\chi_{8,32}\left(s_{2}\right)=\chi_{16,16}\left(s_{2}\right)=\chi_{32,8}\left(s_{2}\right)=4 .
\end{gathered}
$$

- Шифрсистема FOX. Цикловая структура s-бокса есть $\left[1^{1}, 2^{2}, 8^{2}, 21^{1}, 94^{1}, 120^{1}\right]$. Имеем

$$
\begin{gathered}
\chi_{2,128}(s)=\chi_{4,64}(s)=\chi_{8,32}(s)=\chi_{16,16}(s)=\chi_{64,4}(s)=2, \\
\chi_{32,8}(s)=\chi_{128,2}(s)=0 .
\end{gathered}
$$

\section{Список литературы}

1. Бабаш А. В., Шанкин Г.П., Криптография. СОЛОН-Р, Москва, 2002.

2. Бабаш А. В., Криптографические и теоретико-автоматные аспекты современной защиты информации. МЭСИ, Москва, 2010.

3. Погорелов Б. А., Пудовкина М. А., Факторструктуры преобразований. Математические вопросы криптографии (2012) 3, №3, 81-104.

4. Погорелов Б. А., Пудовкина М. А., О расстояниях от подстановок до импримитивных групп при фиксированной системе импримитивности. Дискретная математика (2013) 25, №3, 78-95.

5. Глухов М. М., Елизаров В. П., Нечаев А. А., Алгебра. Гелиос АРВ, Москва, 2003.

6. Панасенко С., Алгоритмы шифрования. Специальный справочник. БХВ-Петербург, Санкт-Петербург, 2009.

7. Barreto P., Rijmen V., The Whirlpool hashing function, First open NESSIE Workshop (2000) 13-14.

8. Specification of SMS4, Block Cipher for WLAN Products - SMS4 (in Chinese).

Статья поступила 25.07.2013. 